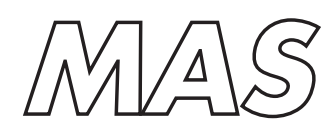

Modelling, Analysis and Simulation

D. Lahaye, P.W. Hemker 
Centrum voor Wiskunde en Informatica (CWI) is the national research institute for Mathematics and Computer Science. It is sponsored by the Netherlands Organisation for Scientific Research (NWO).

CWI is a founding member of ERCIM, the European Research Consortium for Informatics and Mathematics.

CWI's research has a theme-oriented structure and is grouped into four clusters. Listed below are the names of the clusters and in parentheses their acronyms.

Probability, Networks and Algorithms (PNA)

Software Engineering (SEN)

\section{Modelling, Analysis and Simulation (MAS)}

Information Systems (INS)

Copyright (C) 2007, Stichting Centrum voor Wiskunde en Informatica

P.O. Box 94079, 1090 GB Amsterdam (NL)

Kruislaan 413, 1098 SJ Amsterdam (NL)

Telephone +31205929333

Telefax +31205924199

ISSN 1386-3703 


\title{
An $h p$-adaptive strategy for the solution of the exact kernel curved wire Pocklington equation
}

\author{
ABSTRACT \\ In this paper we introduce an adaptive method for the numerical solution of the Pocklington \\ integro-differential equation with exact kernel for the current induced in a smoothly curved thin \\ wire antenna. The $h p$-adaptive technique is based on the representation of the discrete solution, \\ which is expanded in a piecewise $p$-hierarchical basis. The key element in the strategy is an \\ element-by-element criterion that controls the $h$ - or $p$-refinement. Numerical results demonstrate \\ both the simplicity and efficiency of the approach.
}

2000 Mathematics Subject Classification: 45J05, 65N30, 41A10, 78A50

Keywords and Phrases: electromagnetic scattering; wire antenna; Pocklington; finite element approximations; $h p-$ adaptivity

Note: This research is supported by the Dutch Ministry of Economic Affairs through the project IOP-EMVT 04302. 



\title{
AN $h p$-ADAPTIVE STRATEGY FOR THE SOLUTION OF THE EXACT KERNEL CURVED WIRE POCKLINGTON EQUATION
}

\author{
D. LAHAYE* AND P. W. HEMKER*
}

\begin{abstract}
In this paper we introduce an adaptive method for the numerical solution of the Pocklington integro-differential equation with exact kernel for the current induced in a smoothly curved thin wire antenna. The $h p$-adaptive technique is based on the representation of the discrete solution, which is expanded in a piecewise $p$-hierarchical basis. The key element in the strategy is an element-by-element criterion that controls the $h$ - or $p$-refinement. Numerical results demonstrate both the simplicity and efficiency of the approach.
\end{abstract}

Key words. electromagnetic scattering, wire antenna, Pocklington, exact kernel, finite element approximations, $h p$-adaptivity.

AMS subject classifications. 45J05, 65N30, 41A10, 78A50.

1. Introduction. In this study we treat electric field scattering from thin curved wire antennas. The current that an incident electrical field induces in the antenna is computed by solving the Pocklington integro-differential equation $[12,7,15]$. In engineering literature the reduced kernel approximation is typically used. However, if very fine meshes are used for the discretization, the ill-posedness of the resulting problem causes spurious oscillations in the numerical solution, which prevents the computation of highly accurate solutions $[8,2,13,14,16]$. We therefore treat the computationally more challenging exact kernel model $[4,3]$.

The finite element (FE) technique proposed in this paper achieves high accuracy at moderate computational cost by automatic adaption of both the mesh width $(h$ adaptation) and the polynomial degree ( $p$-adaptation) of the approximation to the local smoothness of the solution. The discrete solution is expanded in a piecewise hierarchical basis [18], consisting of the standard linear FE shape functions enriched with higher order bubble functions. Apart from the treatment of a smooth arbitrarily curved wire, a new aspect in this work is the element-by-element criterion for $h$ - or $p$ refinement. As shown below, this criterion is based on the behaviour of the coefficients of the discrete solution in the hierarchical basis representation.

The fact that we want to solve the problem for an arbitrarily curved wire geometries implies that the resulting discrete system generally will not be of Toeplitz type. Such geometries prevent the use of the corresponding computational shortcuts that makes the computation for linear, circular and helical antennas more efficient [9].

This paper is structured as follows: in Section 1 we introduce the Pocklington integro-differential equation and its Bubnov-Galerkin discretization. In Section 2 we describe our approximation of the exact kernel, the choice of the finite element basis functions and the $h p$-adaptive strategy. In Section 3 we describe the construction of the discrete operators and the $h p$-adaptive algorithm. In Section 4 we provide evidence of the effectiveness of our approach and finally we summarize the main conclusions of this work.

* Centrum voor Wiskunde en Informatica (CWI), P.O. Box 94079, 1090 GB Amstersdam, The Netherlands (d.lahaye@cwi.nl, p.w.hemker@cwi.nl). This research is supported by the Dutch Ministry of Economic Affairs through the project IOP-EMVT 04302. 


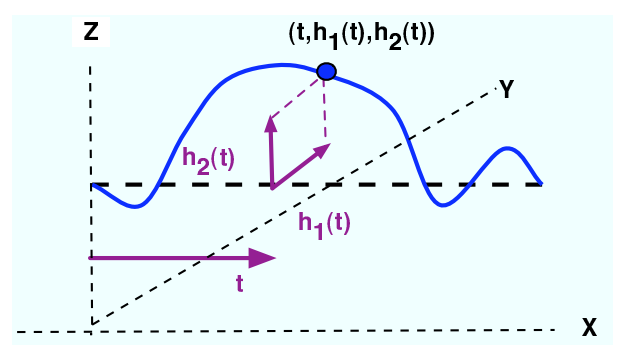

FIG. 1.1. Curved wire geometry.

1.1. Pocklington's equation for the curved thin wire antenna. The geometry of the cylindrical smoothly curved wire is described by the location of its axis $\left(t, h_{1}(t), h_{2}(t)\right) \in \mathbb{R}^{3}$ and its radius $a$. Here, parametrized by $t, h_{1}(t)$ and $h_{2}(t)$ describe the horizontal and vertical deviations from the straight antenna aligned with the $x$-axis. The hull of the wire is described by $\mathbf{r}(t, \theta, a)=\left(t, h_{1}(t), h_{2}(t)\right)+$ $a(0, \cos \theta, \sin \theta)^{1}$. On the wire axis we denote its direction by

$$
\mathbf{s}(t)=\frac{\partial \mathbf{r}(t, \theta, 0) / \partial t}{\|\partial \mathbf{r}(t, \theta, 0) / \partial t\|} .
$$

As shorthand we use $\mathbf{r}=\mathbf{r}(t, \theta, a), \mathbf{z}=\mathbf{r}\left(t^{\prime}, \theta^{\prime}, a\right)$ and $\mathbf{s}=\mathbf{s}(t)$.

We consider the current on the cylindrical hull $\mathbf{r}(t, \theta, a)$ to be caused by either an incoming plane wave electromagnetic field or an impressed current source over a finite gap in case of a receiving or an emitting wire, respectively. We use the simplifying approximation that the current only exists on the lateral surface of the wire, i.e., $\mathbf{s} \cdot \mathbf{J}_{\mathbf{S}}(\mathbf{r}(t, \theta, a)) \approx I(t, \theta) /(2 \pi a)$, where $\mathbf{J}_{\mathbf{S}}$ is the surface current density and $I(t, \theta)$ the total current over the wire. The derivative of the current in the direction of the wire is denoted by $I^{\prime}(t, \theta)=\frac{\partial}{\partial t} I(t, \theta)=\left(\mathbf{s} \cdot \nabla_{\mathbf{r}}\right) I(t, \theta)$.

In case of a receiving antenna, Pocklington's integral equation describing the relation between the electric field $\mathbf{E}^{i n}$ and the current $I(t, \theta)$ reads (cfr. $[12,7,15]$ )

$$
\begin{aligned}
-\imath \omega \varepsilon \mathbf{s} \cdot \mathbf{E}^{i n}(\mathbf{r})= & k^{2} \int_{t^{\prime}} \int_{\theta^{\prime}} \mathbf{s} \cdot \mathbf{s}^{\prime} \frac{I\left(t^{\prime}, \theta^{\prime}\right)}{2 \pi} G(\|\mathbf{r}-\mathbf{z}\|) d t^{\prime} d \theta^{\prime} \\
& +\left(\mathbf{s} \cdot \nabla_{\mathbf{r}}\right) \int_{t^{\prime}} \int_{\theta^{\prime}} \frac{\frac{\partial}{\partial t^{\prime}} I\left(t^{\prime}, \theta^{\prime}\right)}{2 \pi} G(\|\mathbf{r}-\mathbf{z}\|) d t^{\prime} d \theta^{\prime}
\end{aligned}
$$

where

$$
G(r)=\frac{e^{-\imath k r}}{4 \pi r}
$$

This is the equation with exact kernel. The emitting antenna is modeled by setting $\mathbf{E}^{i n}(\mathbf{r}) \equiv 0$ and impressing a given current $I(t)=I_{0}$ on the part of the integration domain that corresponds with the gap in the antenna.

\footnotetext{
${ }^{1}$ Notice that bending a circular cylindrical antenna results in an antenna that is no longer circular cylindrical.
} 
For the discretization we write this equation in its weak formulation, using a weighting function $\psi(t, \theta)$ and integrating over the wire hull

$$
\begin{aligned}
& -2 \pi \imath \omega \varepsilon \int_{t} \int_{\theta} \psi(t, \theta) \mathbf{s} \cdot \mathbf{E}^{i n}(\mathbf{r}) d t d \theta \\
= & k^{2} \int_{t} \int_{\theta} \int_{t^{\prime}} \int_{\theta^{\prime}} \psi(t, \theta) I\left(t^{\prime}, \theta^{\prime}\right) \mathbf{s} \cdot \mathbf{s}^{\prime} G(\|\mathbf{r}-\mathbf{z}\|) d t^{\prime} d \theta^{\prime} d t d \theta \\
& -\int_{t} \int_{\theta} \int_{t^{\prime}} \int_{\theta^{\prime}} \psi^{\prime}(t, \theta) \frac{\partial I\left(t^{\prime}, \theta^{\prime}\right)}{\partial t^{\prime}} G(\|\mathbf{r}-\mathbf{z}\|) d t^{\prime} d \theta^{\prime} d t d \theta .
\end{aligned}
$$

We remember that $k^{2}=\omega^{2} / c^{2}=\omega^{2} \mu \varepsilon$ and find

$$
\|\mathbf{r}-\mathbf{z}\|=\sqrt{\begin{array}{r}
\left(t-t^{\prime}\right)^{2}+\left(h_{1}(t)-h_{1}\left(t^{\prime}\right)+2 a \sin \alpha \sin \beta\right)^{2} \\
+\quad\left(h_{2}(t)-h_{2}\left(t^{\prime}\right)-2 a \cos \alpha \sin \beta\right)^{2}
\end{array}}
$$

with $\alpha=\left(\theta+\theta^{\prime}\right) / 2$ and $\beta=\left(\theta^{\prime}-\theta\right) / 2$.

1.2. The Galerkin discretization. In the discrete approximation we neglect the possible dependence of $I(t, \theta)$ on $\theta$ and we set

$$
I(t, \theta)=\sum_{j} I_{j} \phi_{j}(t)
$$

Note that in this discrete form we do not represent the possible $\theta$-dependence of $I(t, \theta)$. This results in a formalism similar to the one analysed in [16]. Similarly to (1.4) we take for the weighting functions $\psi(t, \theta)=\phi_{i}(t)$ to obtain the Bubnov-Galerkin discretization

$$
\begin{aligned}
& 2 \pi \imath \omega \varepsilon \int_{t} \int_{\theta} \phi_{i}(t) \mathbf{s} \cdot \mathbf{E}^{i n}(\mathbf{r}) d t d \theta=\sum_{j} I_{j} \\
& \left(-k^{2} \int_{t} \int_{\theta} \int_{t^{\prime}} \int_{\theta^{\prime}} \phi_{i}(t) \phi_{j}\left(t^{\prime}\right) \mathbf{s} \cdot \mathbf{s}^{\prime} G(\|\mathbf{r}-\mathbf{z}\|) d t^{\prime} d \theta^{\prime} d t d \theta\right. \\
& \left.\quad+\int_{t} \int_{\theta} \int_{t^{\prime}} \int_{\theta^{\prime}} \phi_{i}^{\prime}(t) \phi_{j}^{\prime}\left(t^{\prime}\right) G(\|\mathbf{r}-\mathbf{z}\|) d t^{\prime} d \theta^{\prime} d t d \theta\right) .
\end{aligned}
$$

Thus, in order to compute the current $I(t)$, we have to solve the symmetric linear system

$$
\sum_{j}\left(B_{i j}-k^{2} A_{i j}\right) I_{j}=4 \pi^{2} \imath \omega \varepsilon f_{i}
$$

where $f_{i}$ is given by

$$
f_{i}=\frac{1}{2 \pi} \int_{t} \int_{\theta} \phi_{i}(t) \mathbf{s} \cdot \mathbf{E}^{i n}(\mathbf{r}) d t d \theta=\int_{t} \phi_{i}(t)<\mathbf{s} \cdot \mathbf{E}^{i n}>(t) d t,
$$

where $<\mathbf{s} \cdot \mathbf{E}^{i n}>(t)$ denotes

$$
<\mathbf{s} \cdot \mathbf{E}^{i n}>(t)=\frac{1}{2 \pi} \int_{\theta} \mathbf{s} \cdot \mathbf{E}^{i n}(\mathbf{r}) d \theta
$$


and where the impedance matrices $A$ and $B$ corresponding to the vector and scalar potential are defined by

$$
A_{i j}=\int_{t} \int_{t^{\prime}} \mathbf{s} \cdot \mathbf{s}^{\prime} \phi_{i}(t) \phi_{j}\left(t^{\prime}\right) \widetilde{I_{G}}\left(t, t^{\prime}\right) d t^{\prime} d t
$$

and

$$
B_{i j}=\int_{t} \int_{t^{\prime}} \phi_{i}^{\prime}(t) \phi_{j}^{\prime}\left(t^{\prime}\right) \widetilde{I_{G}}\left(t, t^{\prime}\right) d t^{\prime} d t
$$

respectively, where

$$
\widetilde{I_{G}}\left(t, t^{\prime}\right)=\int_{\theta} \int_{\theta^{\prime}} G(\|\mathbf{r}-\mathbf{z}\|) d \theta^{\prime} d \theta
$$

If the wire is smoothly curved (i.e., if the radius of curvature is much larger than the wire thickness) we see that $\widetilde{I_{G}}\left(t, t^{\prime}\right)$ is well approximated by $[13$, eq. (1)]

$$
I_{G}(\zeta, \gamma)=2 \int_{\beta=0}^{\pi / 2} \frac{e^{-\imath \gamma \sqrt{\zeta^{2}+4 \sin ^{2} \beta}}}{\sqrt{\zeta^{2}+4 \sin ^{2} \beta}} d \beta
$$

with $\zeta=\frac{1}{a} \sqrt{\Delta t^{2}+\Delta h_{1}^{2}+\Delta h_{2}^{2}}=\frac{1}{a} \sqrt{\left(t-t^{\prime}\right)^{2}+\left(h_{1}(t)-h_{1}\left(t^{\prime}\right)\right)^{2}+\left(h_{2}(t)-h_{2}\left(t^{\prime}\right)\right)^{2}}$ and $\gamma=k a$. This means that $\gamma$ denotes the wave-number scaled to the wire radius and $\zeta$ a scaled distance between two points on the wire. To account for the curvature, we later also need the notation $\mathfrak{h}=a \zeta / \Delta t$. As we are interested in antennas with a length of the order of $1 \mathrm{~m}$, a radius of about $0.001 \mathrm{~m}$, and frequencies in the order of $1 \mathrm{GHz}$, interesting values of $\gamma$ range from about 0.005 to about 0.02 . In principle, the technique explained in this paper can be applied to any range of $\gamma$. However, different ranges will require other expansions of the form (2.8) and (2.9).

1.3. Functional of the solution. We are particularly interested in the accurate computation of the voltage $V$ induced over a finite gap in a receiving antenna. This voltage is computed by application of the reciprocity theorem to the antenna in an emitting and a receiving state. Denoting by $I(t)$ the current induced in the emitting antenna with constant current $I_{0}$ impressed over the gap, and by $\left\langle\mathbf{s} \cdot \mathbf{E}^{i n}(t)>\right.$ the quantity introduced in (1.7), the induced voltage is equal to (see e.g., $[5,10,6]$ )

$$
V=\int_{t} \frac{I(t)}{I_{0}}<\mathbf{s} \cdot \mathbf{E}^{i n}>(t) d t
$$

where the integral is computed over the antenna, excluding the gap.

2. Numerical Method. In this section we show three basic techniques that underly our algorithm for the solution of Pocklington's equation: (1) the approximation of the exact kernel, (2) the introduction of a local $p$-hierarchical finite-element basis, and, (3) the principle of the $h p$-adaptive strategy used to determine a sufficiently accurate discretization.

2.1. Approximation of the kernel. An essential point in the efficient evaluation of the impedance matrices (1.8) and (1.9) is the evaluation of $I_{G}(\zeta, \gamma)$. To understand how this function is evaluated, in the discussion below we first distinguish between large and small values for $\zeta$. 
For large values of $\zeta$ we find the approximation

$$
I_{G}(\zeta, \gamma)=\pi \frac{e^{-\imath \gamma \sqrt{2+\zeta^{2}}}}{\sqrt{2+\zeta^{2}}}+e(\zeta, \gamma)
$$

which is much similar to the reduced kernel expression. The relative error in this approximation satisfies, asymptotically for large $\zeta$,

$$
\left|\frac{e(\zeta, \gamma)}{I_{G}(\zeta, \gamma)}\right|=2\left(3+(\gamma \zeta)^{2}\right) \delta^{2} \zeta^{-2}+\mathcal{O}\left(\zeta^{-4}\right)
$$

where $\delta$ denotes the relative deviation of the antenna from the straight line, i.e., $\delta=\sqrt{\Delta h_{1}^{2}+\Delta h_{2}^{2}} /(\zeta a)$. Further, we may assume that the length of the antenna is of the order of one wavelength, i.e., $\gamma \zeta \approx \mathcal{O}(1)$. So, $(2.2)$ describes how the accuracy of the approximation (2.1) depends on $\delta$ and $\zeta$. For $\delta=0$, the approximation (2.1) corresponds with the approximation in $[4$, eq.(3.3)] for the straight antenna. Below we show how (2.1) is used in actual computations for general $\delta$.

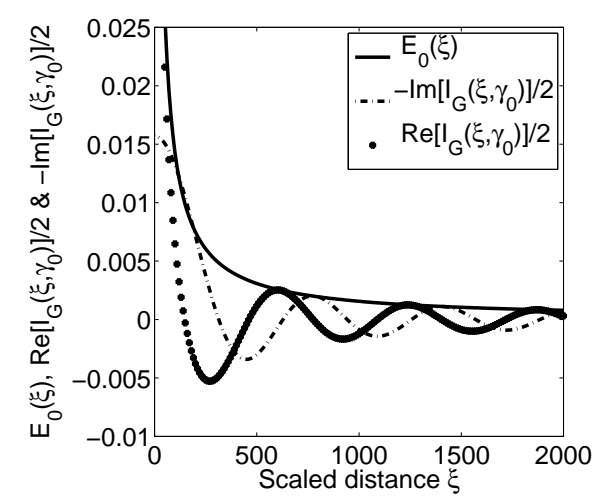

(a) $E_{0}(\zeta), \operatorname{Re}\left[I_{G}\left(\zeta, \gamma_{0}\right)\right]$ and $\operatorname{Im}\left[I_{G}\left(\zeta, \gamma_{0}\right)\right]$

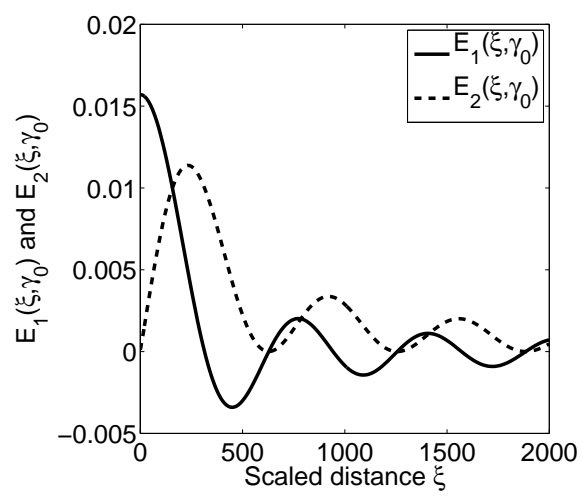

(b) $E_{1}\left(\zeta, \gamma_{0}\right)$ and $E_{2}\left(\zeta, \gamma_{0}\right)$

FIG. 2.1. The functions $E_{0}(\zeta), E_{1}\left(\zeta, \gamma_{0}\right)=-\operatorname{Im}\left[I_{G}\left(\zeta, \gamma_{0}\right)\right] / 2$ and $E_{0}(\zeta)-E_{2}\left(\zeta, \gamma_{0}\right)=$ $\operatorname{Re}\left[I_{G}\left(\zeta, \gamma_{0}\right)\right] / 2$ for $0 \leq \zeta \leq 2000$ and $\gamma_{0}=0.01$.

For small values of $\zeta$ the vanishing denominator in the integrand of (1.11) induces the singularity of the kernel. Therefore we split $I_{G}(\zeta, \gamma)$ in a singular and a regular part. We introduce the real functions $E_{0}(\zeta), E_{1}(\zeta, \gamma)$ and $E_{2}(\zeta, \gamma)$ in order to write

$$
\begin{aligned}
I_{G}(\zeta, \gamma) & =2 \int_{\beta=0}^{\pi / 2} \frac{1}{\sqrt{\zeta^{2}+4 \sin ^{2} \beta}} d \beta+2 \int_{\beta=0}^{\pi / 2} \frac{e^{-\imath \gamma \sqrt{\zeta^{2}+4 \sin ^{2} \beta}}-1}{\sqrt{\zeta^{2}+4 \sin ^{2} \beta}} d \beta \\
& =2 E_{0}(\zeta)-2\left(E_{2}(\zeta, \gamma)+\imath E_{1}(\zeta, \gamma)\right),
\end{aligned}
$$

with for the singular part $[11,17]$

$$
E_{0}(\zeta)=\int_{\beta=0}^{\pi / 2} \frac{1}{\sqrt{\zeta^{2}+(2 \sin \beta)^{2}}} d \beta=\frac{1}{\sqrt{4+\zeta^{2}}} K\left(\frac{4}{4+\zeta^{2}}\right)=\frac{1}{\zeta} K\left(-4 / \zeta^{2}\right)
$$


Here, $K(\zeta)$ is the elliptic integral of the first kind [1]. The function $E_{0}(\zeta)$ is simple to compute (see Figure 2.1). For $\zeta \rightarrow 0$ we have asymptotically

$$
\begin{aligned}
E_{0}(\zeta)= & \frac{1}{2}\left(\log (2)-\log (\zeta)-\psi^{(0)}\left(\frac{1}{2}\right)-\gamma\right) \\
& -\frac{1}{32}\left(\log (2)-\log (\zeta)-\frac{1}{2} \psi^{(0)}\left(\frac{3}{2}\right)-\frac{1}{2} \psi^{(0)}\left(-\frac{1}{2}\right)-\gamma+1\right) \zeta^{2} \\
& +O\left(\zeta^{4}\right)
\end{aligned}
$$

Here (and only here) $\gamma$ is the Euler gamma and $\psi^{(0)}$ is the polygamma function. On the other hand, for $\zeta \rightarrow \infty$ we find asymptotically

$$
\log \left(E_{0}(\zeta)\right)=\log (\pi / 2)-\log (\zeta)-\zeta^{-2}+O\left(\zeta^{-4}\right)
$$

With such a priori knowledge it is a simple matter of elementary numerical analysis to construct an expansion that approximates the function $E_{0}(\zeta)$ up to a required accuracy. In fact we use an expansion in terms $\zeta^{2 i}$ and $\zeta^{2 i} \log \zeta$ with a sufficient number of terms to approximate $E_{0}(\zeta)$ with required accuracy in some neighbourhood of the origin. E.g., $0 \leq i \leq 4$ yields an accuracy of 6 digits on [0,2]. This type of expansion will be used below with higher accuracy for the approximation of $\operatorname{Re}\left[\widetilde{I}_{G}(\zeta, \gamma)\right]$ for small $\zeta$.

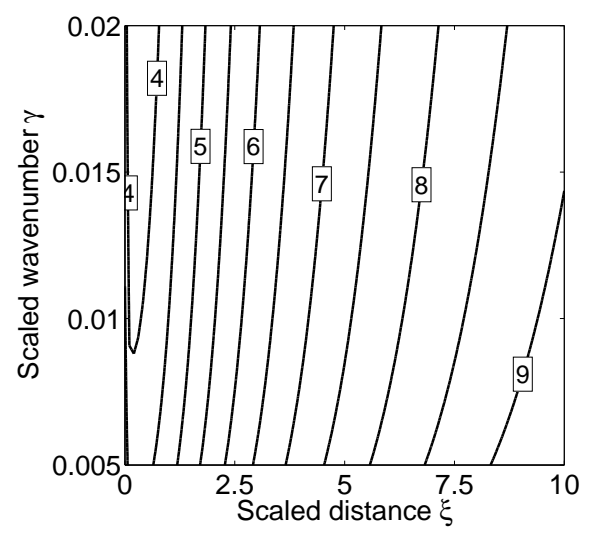

FIG. 2.2. Digits of accuracy in $\widetilde{E}_{2}(\zeta, \gamma)$ for $0 \leq \xi \leq 10$ and $0.005<\gamma<0.02$.

In the non-singular part of $I_{G}(\zeta, \gamma)$ we distinguish the imaginary and the real part

$$
E_{1}(\zeta, \gamma)=\gamma \int_{\beta=0}^{\beta=\pi / 2} \frac{\sin \left(\gamma \sqrt{\zeta^{2}+(2 \sin \beta)^{2}}\right)}{\gamma \sqrt{\zeta^{2}+(2 \sin \beta)^{2}}} d \beta
$$

and

$$
E_{2}(\zeta, \gamma)=\gamma \int_{\beta=0}^{\beta=\pi / 2} \frac{\sin ^{2}\left(\frac{\gamma}{2} \sqrt{\zeta^{2}+(2 \sin \beta)^{2}}\right)}{\frac{\gamma}{2} \sqrt{\zeta^{2}+(2 \sin \beta)^{2}}} d \beta
$$


Studying the asymptotic behaviour of $E_{1}(\zeta, \gamma)$ for $\zeta \rightarrow \infty$ we find an approximation

$$
\begin{gathered}
E_{1}(\zeta, \gamma) \approx \widetilde{E}_{1}(\zeta, \gamma)=\frac{\pi}{8}\left[\left(4+\frac{3}{\left(\zeta^{2}+2\right)^{2}}\right) \frac{\sin \left(\gamma \sqrt{\zeta^{2}+2}\right)}{\sqrt{\zeta^{2}+2}}\right. \\
\left.-\frac{3 \gamma \cos \left(\gamma \sqrt{\zeta^{2}+2}\right)}{\left(\zeta^{2}+2\right)^{2}}-\frac{\gamma^{2} \sin \left(\gamma \sqrt{\zeta^{2}+2}\right)}{\left(\zeta^{2}+2\right)^{3 / 2}}\right]
\end{gathered}
$$

that for $\gamma$ in our range of interest, i.e., $0.005 \leq \gamma \leq 0.02$, is accurate to 13 digits. This accuracy extends over all $\zeta$. The accuracy of this formula deteriorates for larger values of $\gamma$, but the approximation remains useful for values $\gamma \lesssim 0.5$.

For $E_{2}(\zeta, \gamma)$ we find a similar approximation

$$
\begin{gathered}
E_{2}(\zeta, \gamma) \approx \widetilde{E}_{2}(\zeta, \gamma)=\frac{\pi}{8}\left[\left(4+\frac{3}{\left(\zeta^{2}+2\right)^{2}}\right) \frac{2 \sin ^{2}\left(\frac{\gamma}{2} \sqrt{\zeta^{2}+2}\right)}{\left(\zeta^{2}+2\right)^{1 / 2}}\right. \\
\left.-\frac{3 \gamma \sin \left(\gamma \sqrt{\zeta^{2}+2}\right)}{\left(\zeta^{2}+2\right)^{2}}+\frac{\gamma^{2} \cos \left(\gamma \sqrt{\zeta^{2}+2}\right)}{\left(\zeta^{2}+2\right)^{3 / 2}}\right] .
\end{gathered}
$$

However, this approximation is less accurate. In our range of interest $0.005 \leq \gamma \leq 0.02$ the accuracy is only 3.5 digits. The error becomes more significant only for the smaller values of $\zeta$ (see Figure 2.2). So, in order to realize an efficient and accurate evaluation for the real part of $I_{G}(\zeta, \gamma)$, we construct an approximation combined with $E_{0}(\zeta)$. Because of the singularity at $\zeta=0$, for small values of $\zeta$ we use four terms from the asymptotic expansion for $\zeta \rightarrow 0$ of $E_{0}(\zeta)$, and we correct this with a multivariate approximation of the difference between $E_{0}(\zeta)-E_{2}(\zeta, \gamma)$ and this asymptotic expression. For the larger values of $\zeta$ we take $(2.7)$ and we correct it with a series expansion in odd powers of $1 / \zeta$. Combining the expressions $(2.5),(2.6)$ and (2.7) it is easily verified that in the limit for large $\zeta$ our approximation for $I_{G}$ yields the modified reduced kernel expression (2.1).

Summarizing, for $\widetilde{I}_{G}(\zeta, y)$, the approximation of $I_{G}(\zeta, \gamma)$, we find

$$
\begin{aligned}
& \operatorname{Im}\left[\widetilde{I}_{G}(\zeta, \gamma)\right]=\widetilde{E}_{1}(\zeta, \gamma) \\
& \operatorname{Re}\left[\widetilde{I}_{G}(\zeta, \gamma)\right] \in\left\{\begin{array}{c}
\operatorname{Span}\left\{\zeta^{2 i} \gamma^{j}(\log \zeta)^{k} ; i=0, \cdots, 6, j=0,1,2, k=0,1\right\} \\
\text { for } 0<\zeta<3.1, \\
\widetilde{E}_{2}(\zeta, \gamma)+\operatorname{Span}\left\{\zeta^{-4 i+1} \gamma^{j} ; i=0,1, \cdots, 5, j=0,1\right\}+ \\
+\operatorname{Span}\left\{\begin{array}{c}
;=0,1, \cdots, 4\} \\
\zeta^{-4 i+3} ; i=3.1,
\end{array}\right.
\end{array}\right.
\end{aligned}
$$

where the coefficients in the expansion (2.9) are determined as to obtain the best least squares fit with $\operatorname{Re}\left[I_{G}(\zeta, \gamma)\right]$. In the range of interest the accuracy of the approximation is at least 8 digits.

Splitting off the singular part for the evaluation of the integrals. In order to compute the discrete operator entries (1.8)-(1.9) involving integrals of our singular kernel function $I_{G}$, we split this function in a singular and a regular part. That is, we write

$$
I_{G}(\zeta, \gamma)=I_{S}(\zeta, \gamma)+I_{R}(\zeta, \gamma)
$$


where both $I_{S}(\zeta, \gamma)$ and $I_{R}(\zeta, \gamma)$ are smooth functions and where $I_{S}(\zeta, \gamma)$ contains the logarithmic terms and $I_{S}(\zeta, \gamma)=0$ for $\zeta>\zeta_{0}$ for some $\zeta_{0}$. Integrals involving $I_{S}(\zeta, \gamma)$ and $I_{R}(\zeta, \gamma)$ are computed analytically and by quadrature, respectively. In order to assure that both $I_{S}(\zeta, \gamma)$ and $I_{R}(\zeta, \gamma)$ are smooth, we take $I_{S}(\zeta, \gamma)=I_{G}(\zeta, \gamma)-$ $I_{\text {Taylor }}(\zeta, \gamma)$ for $\zeta \leq \zeta_{0}$, where $I_{\text {Taylor }}(\zeta, \gamma)$ is a Taylor expansion of $I_{G}$ in $\zeta$ at $\zeta=\zeta_{0}$. The part $I_{R}$ is the smooth remaining part, defined by (2.10). For the numerical approximations $\widetilde{I}_{S}$ and $\widetilde{I}_{R}$ we take $\zeta_{0}=3.1$ and obtain

$$
\begin{aligned}
& \widetilde{I}_{S}(\zeta, \gamma) \in \operatorname{Span}\left\{\zeta^{2 i} \gamma^{j}(\log \zeta)^{k} ; i=0, \cdots, 6, j=0,1,2, k=0,1\right\} \\
& \text { for } 0<\zeta<3.1 \text {, }
\end{aligned}
$$

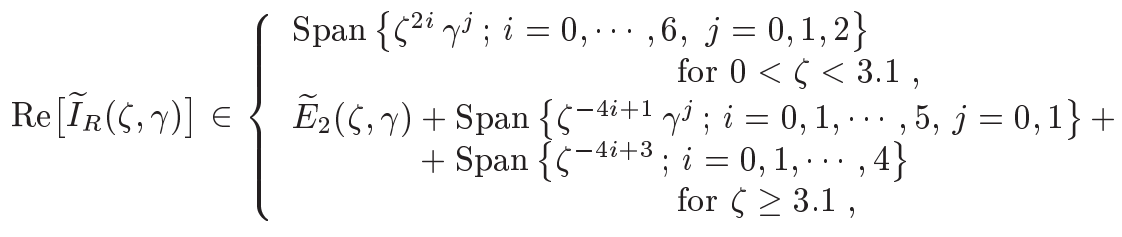

$$
\begin{aligned}
& \operatorname{Im}\left[\widetilde{I}_{R}(\zeta, \gamma)\right]=\widetilde{E}_{1}(\zeta, \gamma)
\end{aligned}
$$

2.2. Choice of the local basis. In order to form the discrete system we have to make a choice for the basis functions $\left\{\phi_{j}\right\}$ in (1.5). Because we want to construct an $h p$-adaptive method to obtain optimal efficiency, we partition $\Omega$, the domain of integration for $t$ and $t^{\prime}$, in elements of arbitary sizes. So we obtain the mesh

$$
t_{0}<t_{1}<t_{2}<\cdots<t_{N}
$$

or

$$
\Omega_{\Delta}=\bigcup_{i=1}^{N}\left[t_{i-1}, t_{i}\right]=\bigcup_{i=1}^{N} \Delta_{i} .
$$

On this mesh we introduce a continuous, piecewise polynomial basis of degree $p$ with piecewise linear functions and higher order hierarchical bubble functions (i.e., $p \geq 1$ ), so that the basis for our approximation on the interval $\Delta_{i}=\left[t_{i-1}, t_{i}\right]$ becomes

$$
\begin{aligned}
& \phi_{i, 0}(t)=\phi_{0+(i-1) p}(t)=\left(t_{i}-t\right) /\left(t_{i}-t_{i-1}\right) \\
& \phi_{i, k}(t)=\phi_{k+(i-1) p}(t)=\prod_{j=1}^{k+1} \frac{t-t_{i, j, k+1}}{\left(t_{i}-t_{i-1}\right)^{k+1}} \text { for } k=1, \cdots, p-1, \\
& \phi_{i, p}(t)=\phi_{p+(i-1) p}(t)=\left(t-t_{i-1}\right) /\left(t_{i}-t_{i-1}\right),
\end{aligned}
$$

where $t_{i, j, k}=\frac{1+\xi_{j, k}}{2}\left(t_{i}-t_{i-1}\right)+t_{i-1}$ with $\xi_{j, k}$ the $j$-th point in the $k+1$-point Lobatto quadrature rule. In this way we form a piecewise polynomial function space of degree $p$. Notice that all basis functions have support $\Delta_{i}$ except the piecewise linear $\phi_{i p}$, $i=1, \cdots, N-1$, that have support $\Delta_{i} \cup \Delta_{i+1}$. In case that $p$ is varying across elements, the global numbering of the degrees of freedom is modified accordingly. To construct the matrices $A$ and $B$ in (1.8)-(1.9) it is convenient to introduce a local basis for the standard interval $[-1,+1]$ from which the functions $(2.14)$ can be derived. For 
example, for $p=7$, these basis functions are given by

$$
\begin{aligned}
\varphi_{0}(\xi)= & (1-\xi) / 2 \\
\varphi_{1}(\xi)= & (\xi+1)(\xi-1) / 2^{2}, \\
\varphi_{2}(\xi)= & (\xi+1) \xi(\xi-1) / 2^{3} \\
\varphi_{3}(\xi)= & (\xi+1)\left(\xi+\sqrt{\frac{1}{5}}\right)\left(\xi-\sqrt{\frac{1}{5}}\right)(\xi-1) / 2^{4} \\
\varphi_{4}(\xi)= & (\xi+1)\left(\xi+\sqrt{\frac{3}{7}}\right) \xi\left(\xi-\sqrt{\frac{3}{7}}\right)(\xi-1) / 2^{5} \\
\varphi_{5}(\xi)= & (\xi+1)\left(\xi+\sqrt{\frac{1}{21}(7+2 \sqrt{7})}\right)\left(\xi+\sqrt{\frac{1}{21}(7-2 \sqrt{7})}\right) \\
& \left(\xi-\sqrt{\frac{1}{21}(7-2 \sqrt{7})}\right)\left(\xi-\sqrt{\frac{1}{21}(7+2 \sqrt{7})}\right)(\xi-1) / 2^{6}, \\
\varphi_{6}(\xi)= & (\xi+1)\left(\xi+\sqrt{\frac{1}{33}(15+2 \sqrt{15})}\right)\left(\xi+\sqrt{\frac{1}{33}(15-2 \sqrt{15})}\right) \xi \\
& \left(\xi+\sqrt{\frac{1}{33}(15-2 \sqrt{15})}\right)\left(\xi-\sqrt{\frac{1}{33}(15+2 \sqrt{15})}\right)(\xi-1) / 2^{7}, \\
\varphi_{p}(\xi)= & (\xi+1) / 2 .
\end{aligned}
$$

With the basis function introduced in (2.15), the solution on element $\Delta_{i}$ is approximated by $\sum_{k=0}^{p} c_{k} \phi_{i, k}$.

2.3. The principle of the $h p$-adaptive strategy. The $h p$-adaptive strategy in this paper relies on the above $p$-hierarchical base and makes use of the fact that for smooth functions the coefficients -on a single element in a piecewise polynomial approximation- are supposed to decrease as a geometric sequence.

More precisely, we can say that the approximation of a $C^{p+1}$-function in a neighbourhood of width $2 h$ allows a $p$-hierarchical polynomial representation in which the coefficients are bounded by a geometrically decreasing sequence, if $h$ is small enough. This is easily seen for a local basis with functions $\left\{t^{i}\right\}_{i=0, \ldots, p}$ for which the terms in the Taylor series expansion are bounded by $h^{i}\|f\|_{C^{p}} / i !, i=0, \ldots, p$. For the basis (2.15), we find for the coefficients

$$
\begin{aligned}
\left|c_{p}+c_{0}\right| / 2 & \leq \quad\left|f_{0}+h^{2}\left(f_{2}+h^{2}\left(f_{4}+h^{2} f_{6}\right)\right)\right| \\
\left|c_{p}-c_{0}\right| / 2 & \leq h\left|f_{1}+h^{2}\left(f_{3}+h^{2}\left(f_{5}+h^{2} f_{7}\right)\right)\right| \\
\left|c_{1}\right| & \leq h^{2}\left|f_{2}+h^{2}\left(\frac{6}{5} f_{4}+h^{2} \frac{9}{7} f_{6}\right)\right| \\
\left|c_{2}\right| & \leq h^{3}\left|f_{3}+h^{2}\left(\frac{10}{7} f_{5}+h^{2} \frac{5}{3} f_{7}\right)\right| \\
\left|c_{3}\right| & \leq h^{4}\left|f_{4}+h^{2} \frac{5}{3} f_{6}\right| \\
\left|c_{4}\right| & \leq h^{5}\left|f_{5}+h^{2} \frac{21}{11} f_{7}\right| \\
\left|c_{5}\right| & \leq h^{6}\left|f_{6}\right| \\
\left|c_{6}\right| & \leq h^{7}\left|f_{7}\right|
\end{aligned}
$$

with $f_{i}=f^{(i)}(0) / i$ ! $, i=0, \ldots, p$. This shows that, for small $h$, our hierarchical basis the coefficients $c_{i}, i=1, \ldots, p-1$, are of order $O\left(h^{i+1}\right)$, and bounded by

$$
\left|c_{i}\right| \leq C h^{i+1}\|f\|_{C^{\infty}[-h, h]} .
$$

This not only shows that -for a fine enough mesh- we expect the coefficients for each interval to decrease geometrically, but also that the rate of decrease doubles when the mesh is halved. By the nature of the hierarchical representation it makes sense to consider the last non-vanishing term in the representation as an estimate for the local error. 
The above observations lead to the following $h p$-adaptive strategy. On a (nonuniform) mesh we determine in each interval a $p$-hierarchical representation of the solution with a sufficient number of terms. On each interval the convergence of the approximation is studied. If the rate of decrease of the coefficients is fast enough, then the series is truncated such that an a-priori given tolerance criteria is satisfied. If the rate of decrease is not fast enough, then the interval is split into two smaller intervals of equal size and a new approximation is computed. The process will stop after a finite number of iterations, except in those areas where derivatives are unbounded. At such singular locations the process can be stopped by introducing a limit for the minimal allowed mesh-size. Details of the strategy are explained in Section 3.4.

In this way a sufficiently accurate approximation is obtained, except at welldetermined locations where the solution has a singular behaviour.

The square root singularity. From analysis [13] it is known that at the end-points of a receiving straight-line thin-wire antenna the current shows a square-root singularity. Hence, it is interesting to study the behaviour of the coefficients in the hierarchical representation in this particular case where derivatives become unbounded.

Therefore, we consider the segment of the antenna where the singularity occurs (i.e., near an end-point). Knowing the type of singularity, we can estimate the coefficients in the hierarchical expansion. Denoting the length of the element by $h$, on the elements $[0, h]$ and $[h, 2 h]$ we approximate the function $f(t)=\sqrt{t}$ by a such a polynomial approximation. Therefore we fix the values at the endpoints to determine the linear approximation, whereas the higher order contributions are determined by optimal $L^{2}$-approximation. Because the functions $\varphi_{k}$ are not $L^{2}$-orthogonal, the coefficients (slightly) depend on the order of the approximation. A simple computation shows that all coefficients are of order $\mathcal{O}(\sqrt{h})$, and that on the first interval $[0, h]$ the coefficients increase for the higher order terms. On the second interval $[h, 2 h]$ they decrease by several orders of magnitude. In Figure 2.3 we show these theoretical coefficients on a logarithmic scale for approximations upto degree 7 . It is obvious that on the interval $[0, h] h$-refinement rather than $p$-refinement is the proper strategy to obtain better accuracy, while the contrary is true on the interval $[h, 2 h]$.

The theoretical behavior of the coefficients for the function $f(t)=\sqrt{t}$ is recovered in the computation of the coefficients computed for the current on the receiving straight-line thin-wire antenna. To illustrate this point, we consider the computation of the current in a straight wire of length $L=3 \mathrm{~m}$ and radius $a=0.02 \mathrm{~m}$, induced by a plane wave exitation of wavenumber $k=1 \mathrm{~m}^{-1}$ and propagation vector perpendicular to the antenna axis. We employ an uniform mesh of 128 elements, all containing a local polynomial approximation of degree seven. In Figure 2.4, we plot the coefficients of the $p$-hierarchical decomposition of the current on the first and on the fourteenth element. In the presence of discretization error, we consider the latter to be located at a sufficiently large distance away from the boundary to be representative for the interior of the antenna. Like Figure 2.3, it shows that the coefficients increase for the higher order terms on the element containing the singularity, and that they decrease by several orders of magnitude on the fourteenth element where the solution is smooth. In Section 3.4, we explain how this smoothness indicator is used to implement the $h p$-strategy.

3. Implementation. In this section we describe the construction of the discrete operator and the implementation of the $h p$-adaptive strategy.

3.1. Evaluation of the off-diagonal elements. For the off-diagonal elements in (1.8)-(1.9) with $|i-j|>1$, the singularity of the integrand falls outside the domain 


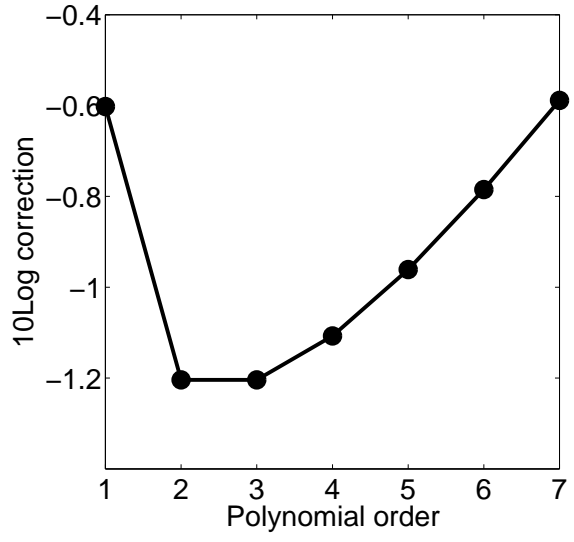

(a) First element.

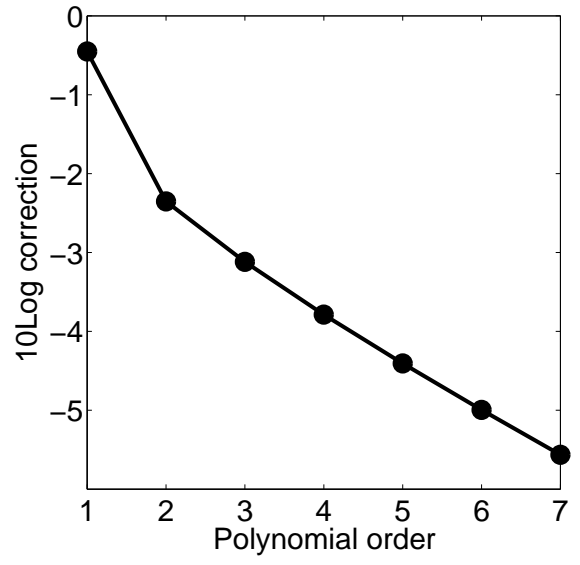

(b) Second element.

FIG. 2.3. Coefficients of the $p$-hierarchical decomposition of $f(t)=\sqrt{t}$ in the element $[0, h]$ and $[h, 2 h]$. The graphs show ${ }^{10} \log \left|c_{k} / h^{\alpha}\right|$ for the coefficients in an approximation of degree $\%$.

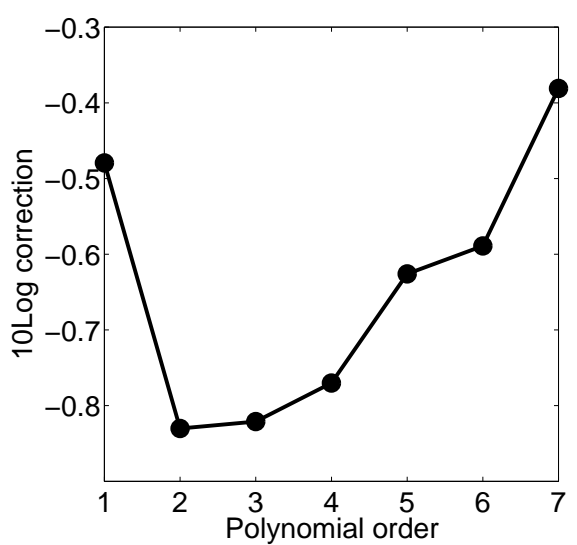

(a) First element.

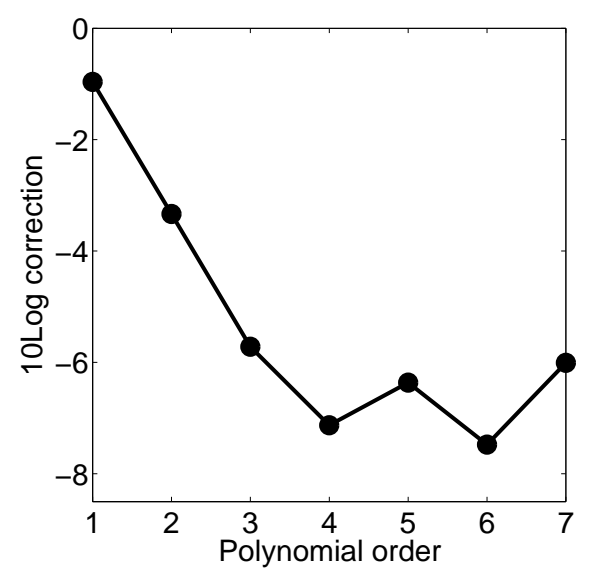

(b) Fourteenth element.

FIG. 2.4. Coefficients of the p-hierarchical decomposition of the computed current on a straightline receiving thin-wire antenna in the first and fourteenth element. The graphs show ${ }^{10} \log \left|c_{k} / h^{\alpha}\right|$ for the coefficients in an approximation of degree $\%$.

of integration, and therefore we can use straightforward Gaussian quadrature. We take a fixed $L$-point Gaussian quadrature rule

$$
\int_{t=a}^{b} f(t) d t \approx(b-a) \sum_{m=1}^{L} w_{m} f\left(t_{m}\right)
$$


and we obtain, with $\Delta t_{i}=t_{i}-t_{i-1}$ and $0 \leq k, l \leq p$,

$$
\begin{aligned}
\left(A_{i, j}\right)_{k, l} & \approx \Delta t_{i} \Delta t_{j} \sum_{m, n=1}^{L} w_{m} \phi_{i, k}\left(t_{i, m}\right) w_{n} \phi_{j, l}\left(t_{j, n}\right)\left(\mathbf{s} \cdot \mathbf{s}^{\prime} \widetilde{I_{G}}\right)\left(t_{i, m}, t_{j, n}\right) \\
& =\Delta t_{i} \Delta t_{j} \sum_{m, n=1}^{L} w_{m} \varphi_{k}\left(t_{m}\right) w_{n} \varphi_{l}\left(t_{n}\right)\left(\mathbf{s} \cdot \mathbf{s}^{\prime} \widetilde{I_{G}}\right)\left(t_{i, m}, t_{j, n}\right), \\
\left(B_{i, j}\right)_{k, l} & \approx \Delta t_{i} \Delta t_{j} \sum_{m, n=1}^{L} w_{m} \phi_{i, k}^{\prime}\left(t_{i, m}\right) w_{n} \phi_{j, l}^{\prime}\left(t_{j, n}\right) \widetilde{I_{G}}\left(t_{i, m}, t_{j, n}\right) \\
& =4 \sum_{m, n=1}^{L} w_{m} \varphi_{k}^{\prime}\left(t_{m}\right) w_{n} \varphi_{l}^{\prime}\left(t_{n}\right) \widetilde{I_{G}}\left(t_{i, m}, t_{j, n}\right) .
\end{aligned}
$$

It is clear that the constant matrices $\Phi$ and $\Phi^{\prime}$, defined by $\Phi_{k m}=w_{m} \varphi_{k}\left(t_{m}\right)$ and $\Phi_{k m}^{\prime}=w_{m} \varphi_{k}^{\prime}\left(t_{m}\right)$, can be computed in advance, so that the work of the construction of the off-diagonal elementary matrices comes down to $\mathcal{O}\left(L^{2}\right)$ evaluations of the function $\widetilde{I_{G}}\left(t, t^{\prime}\right)$. Introducing the matrices $\mathcal{Z}^{i, j}$ and $\widehat{\mathcal{Z}}^{i, j}$ defined by $\mathcal{Z}_{m, n}^{i, j}=$ $I_{G}\left(\zeta\left(t_{i, m}, t_{j, n}\right), \gamma\right)$ and $\widehat{\mathcal{Z}}_{m, n}^{i, j}=\mathbf{s}\left(t_{i, m}\right) \cdot \mathbf{s}^{\prime}\left(t_{j, n}\right) \mathcal{Z}_{m, n}^{i, j}$, respectively, we write the above expressions as

$$
\begin{aligned}
& A_{i, j} \approx \Delta t_{i} \Delta t_{j} \Phi \widehat{\mathcal{Z}}^{i, j} \Phi^{T}, \\
& B_{i, j} \approx 4 \Phi^{\prime} \mathcal{Z}^{i, j}\left(\Phi^{\prime}\right)^{T} .
\end{aligned}
$$

3.2. Evaluation of the diagonal elements. For the diagonal elements in (1.8)-(1.9) with $i=j$, we have to compute integrals of the form

$$
\int_{t=t_{i-1}}^{t=t_{i}} \int_{t^{\prime}=t_{i-1}}^{t^{\prime}=t_{i}} \phi_{i, k}(t) \phi_{i, l}\left(t^{\prime}\right) \chi\left(\left|t-t^{\prime}\right|\right) d t^{\prime} d t
$$

where the function $\chi\left(\left|t-t^{\prime}\right|\right)$ has a singularity at $t=t^{\prime}$ and the distance satisfies $\left|t-t^{\prime}\right|<\Delta t_{i}$. Taking into account the type of dependence of $\chi$ on $t$ and $t^{\prime}$, (see (1.10)) and omitting the factor $\mathbf{s}(t) \cdot \mathbf{s}^{\prime}\left(t^{\prime}\right)$ because of the assumption that the curvature of 
the wire can be neglected over a distance of one or two elements, we approximate

$$
\begin{aligned}
\left(A_{i, i}\right)_{k, l} & =\iint_{t=t_{i-1}, t^{\prime}=t_{i-1}}^{t=t_{i}, t^{\prime}=t_{i}} \phi_{i, k}(t) \phi_{i, l}\left(t^{\prime}\right) \mathbf{s} \cdot \mathbf{s}^{\prime} \widetilde{I_{G}}\left(t, t^{\prime}\right) d t^{\prime} d t \\
& \approx \iint_{t=t_{i-1}, t^{\prime}=t_{i-1}}^{t=t_{i}, t^{\prime}=t_{i}} \phi_{i, k}(t) \phi_{i, l}\left(t^{\prime}\right) \widetilde{I_{G}}\left(t, t^{\prime}\right) d t^{\prime} d t \\
& \approx \iint_{t=t_{i-1}, t^{\prime}=t_{i-1}}^{t=t_{i}, t^{\prime}=t_{i}} \phi_{i, k}(t) \phi_{i, l}\left(t^{\prime}\right) \frac{1}{a} I_{G}\left(\frac{\left|t-t^{\prime}\right| \mathfrak{h}_{i}}{a}, \gamma\right) d t^{\prime} d t \\
& =\frac{1}{a} \iint_{\tau=0, \tau^{\prime}=0}^{\tau=1, \tau^{\prime}=1} \varphi_{k}(\tau) \varphi_{l}\left(\tau^{\prime}\right) I_{G}\left(\frac{\left|\tau-\tau^{\prime}\right| \Delta t_{i} \mathfrak{h}_{i}}{a}, \gamma\right) d\left(\Delta t_{i} \tau^{\prime}\right) d\left(\Delta t_{i} \tau\right) \\
& =\frac{\Delta t_{i}^{2}}{a} \int_{\xi=\frac{-1}{\sqrt{2}}}^{\xi=\frac{1}{\sqrt{2}}} I_{G}\left(|\xi| \sqrt{2} \frac{\Delta t_{i} \mathfrak{h}_{i}}{a}, \gamma\right) \int_{\eta=|\xi|}^{\eta=\sqrt{2}-|\xi|} \varphi_{k}\left(\frac{\eta+\xi}{\sqrt{2}}\right) \varphi_{l}\left(\frac{\eta-\xi}{\sqrt{2}}\right) d \eta d \xi \\
& =\frac{\Delta t_{i}^{2}}{a} \int_{\xi=0}^{\xi=1} \overline{\mathcal{P}}_{k l}(\xi) I_{G}\left(\xi \frac{\Delta t_{i} \mathfrak{h}_{i}}{a}, \gamma\right) d \xi \\
& =\frac{\Delta t_{i}^{2}}{a} \overline{\mathcal{I}}_{k l}\left(\frac{\Delta t_{i} \mathfrak{h}_{i}}{a}, \gamma\right),
\end{aligned}
$$

where $\mathfrak{h}_{\mathfrak{i}}$ is as defined as in Section 1.2, and where the transformations $\tau=(\eta+\xi) / \sqrt{2}$ and $\tau^{\prime}=(\eta-\xi) / \sqrt{2}$ have been used (see Figure 3.1(a)). In (3.4) we introduced the definitions

$$
\begin{aligned}
\widehat{\mathcal{P}}_{k l}(\xi) & =\int_{\eta=|\xi|}^{\eta=\sqrt{2}-|\xi|} \varphi_{k}\left(\frac{\eta+\xi}{\sqrt{2}}\right) \varphi_{l}\left(\frac{\eta-\xi}{\sqrt{2}}\right) d \eta, \\
\overline{\mathcal{P}}_{k l}(\zeta) & =\frac{1}{\sqrt{2}}\left(\widehat{\mathcal{P}}_{k l}\left(\frac{\zeta}{\sqrt{2}}\right)+\widehat{\mathcal{P}}_{k l}\left(\frac{-\zeta}{\sqrt{2}}\right)\right), \\
\overline{\mathcal{I P}}_{k l}(x, \gamma) & =\int_{\xi=0}^{\xi=1} \overline{\mathcal{P}}_{k l}(\xi) I_{G}(\xi x, \gamma) d \xi .
\end{aligned}
$$

For the polynomial base (2.15), the functions $\widehat{\mathcal{P}}_{k l}$ and $\overline{\mathcal{P}}_{k l}$ are also polynomials and they can be computed once and for all. Similarly we find

$$
\left(B_{i, i}\right)_{k, l} \approx \frac{1}{a} \overline{\mathcal{I P}}_{k l}^{\prime}\left(\frac{\Delta t_{i} \mathfrak{h}_{i}}{a}, \gamma\right)
$$

with the polynomials

$$
\begin{aligned}
& {\widehat{\mathcal{P}^{\prime}}}_{k l}(\xi)=\int_{\eta=|\xi|}^{\eta=\sqrt{2}-|\xi|} \varphi_{k}^{\prime}\left(\frac{\eta+\xi}{\sqrt{2}}\right) \varphi_{l}^{\prime}\left(\frac{\eta-\xi}{\sqrt{2}}\right) d \eta, \\
& {\overline{\mathcal{P}^{\prime}}}_{k l}(\zeta)=\frac{1}{\sqrt{2}}\left(\widehat{\mathcal{P}}{ }_{k l}\left(\frac{\zeta}{\sqrt{2}}\right)+{\widehat{\mathcal{P}^{\prime}}}_{k l}\left(\frac{-\zeta}{\sqrt{2}}\right)\right), \\
& \overline{\mathcal{I P}}_{k l}^{\prime}(x, \gamma)=\int_{\xi=0}^{\xi=1} \overline{\mathcal{P}}_{k l}(\xi) I_{G}(\xi x, \gamma) d \xi .
\end{aligned}
$$

To compute $\left(A_{i, j}\right)_{k, l}$ and $\left(B_{i, j}\right)_{k, l}$ we use the splitting (2.10) and take into account that $I_{S}(x, \gamma)$ vanishes for $x>3.1$. This implies that we have to consider two cases: 


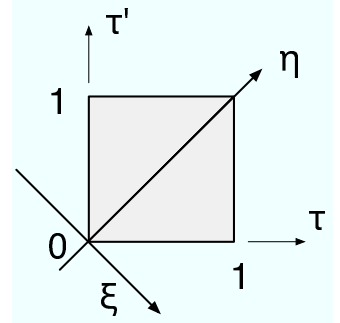

(a) Diagonal element

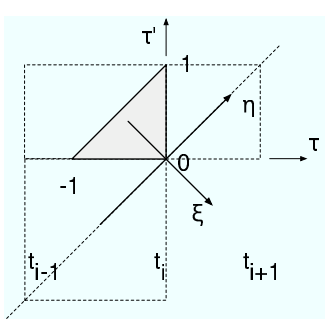

(b) Upper codiagonal element with $\Delta t_{i}>\Delta t_{i+1}$

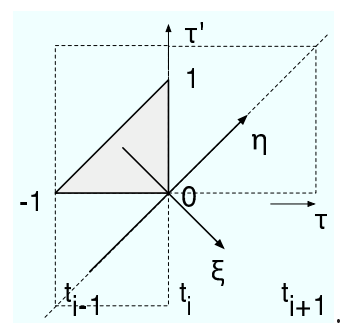

(c) Upper codiagonal element with $\Delta t_{i}>\Delta t_{i+1}$

FIG. 3.1. Coordinate transformation.

the argument $x$ being larger or smaller than 3.1. In the case $x<3.1$, the expressions (3.4) and (3.8) can be implemented directly. However, for $x>3.1$ the discontinuous behaviour ar $x=3.1$ requires a more careful treatment. We get

$$
\begin{aligned}
\overline{\mathcal{I P}}_{k l}(x, \gamma) & =\int_{\xi=0}^{\xi=1} \overline{\mathcal{P}}_{k l}(\xi) I_{S}(x \xi, \gamma) d \xi \\
& =\int_{\xi=0}^{\xi=3.1 / x} \overline{\mathcal{P}}_{k l}(\xi) I_{S}(x \xi, \gamma) d \xi \\
& =\int_{z / x=0}^{z / x=3.1 / x} \overline{\mathcal{P}}_{k l}(z / x) I_{S}(x z / x, \gamma) d z / x \\
& =\frac{1}{x} \int_{z=0}^{z=3.1} \overline{\mathcal{P}}_{k l}(z / x) I_{S}(z, \gamma) d z,
\end{aligned}
$$

and similarly

$$
{\overline{\mathcal{I} \mathcal{P}^{\prime}}}_{k l}(x, \gamma)=\frac{1}{x} \int_{z=0}^{z=3.1} \overline{\mathcal{P}}_{k l}{ }_{k l}(z / x) I_{S}(z, \gamma) d z .
$$

These functions can also be computed beforehand.

3.3. Evaluation of the co-diagonal elements. For the co-diagonal elements in (1.8)-(1.9) we also use the splitting (2.10) of $I_{G}(\zeta, \gamma)$. Due to symmetry, it suffices 
to consider upper co-diagonal elements only. We obtain

$$
\begin{aligned}
\left(A_{i, i+1}\right)_{k, l}= & \iint_{t=t_{i-1}, t^{\prime}=t_{i}}^{t=t_{i}, t^{\prime}=t_{i+1}} \phi_{i, k}(t) \phi_{i, l}\left(t^{\prime}\right) \mathbf{s} \cdot \mathbf{s}^{\prime} \widetilde{I_{G}}\left(t, t^{\prime}\right) d t^{\prime} d t \\
\approx & \int_{t=t_{i-1}}^{t=t_{i}} \int_{t^{\prime}=t_{i}}^{t^{\prime}=t_{i+1}} \phi_{i, k}(t) \phi_{i, l}\left(t^{\prime}\right) \widetilde{I_{G}}\left(t, t^{\prime}\right) d t^{\prime} d t \\
= & \int_{t=t_{i-1}}^{t=t_{i}} \int_{t^{\prime}=t_{i}}^{t^{\prime}=t_{i+1}} \phi_{i, k}(t) \phi_{i, l}\left(t^{\prime}\right) \frac{1}{a} I_{G}\left(\frac{\left|t-t^{\prime}\right| \mathfrak{h}_{i}}{a}, \gamma\right) d t^{\prime} d t \\
= & \int_{t=t_{i-1}}^{t=t_{i}} \int_{t^{\prime}=t_{i}}^{t^{\prime}=t_{i+1}} \phi_{i, k}(t) \phi_{i, l}\left(t^{\prime}\right) \frac{1}{a} I_{R}\left(\frac{\left|t-t^{\prime}\right| \mathfrak{h}_{i}}{a}, \gamma\right) d t^{\prime} d t \\
& +\int_{t=t_{i-1}}^{t=t_{i}} \int_{t^{\prime}=t_{i}}^{t^{\prime}=t_{i+1}} \phi_{i, k}(t) \phi_{i, l}\left(t^{\prime}\right) \frac{1}{a} I_{S}\left(\frac{\left|t-t^{\prime}\right| \mathfrak{h}_{i}}{a}, \gamma\right) d t^{\prime} d t \\
= & \left(A_{i, i+1}^{R}\right)_{k, l}+\left(A_{i, i+1}^{S}\right)_{k, l} .
\end{aligned}
$$

Again we neglect the factor $\mathbf{s} \cdot \mathbf{s}^{\prime}$, assuming that the wire is only mildly curved. The regular part, $A_{i, i+1}^{R}$ is computed by two-dimensional Gaussian quadrature as in Section 3.1. For computing the singular part, $A_{i, i+1}^{S}$, we make use of the fact that $I_{S}(\xi, \gamma)=0$ for $\xi>3.1$ and, in order to be sure that the off-diagonal elements are not influenced by the singularity, we take care that the discretization has no wire segments with ratio $\Delta t_{i} / a$ smaller than 3.1. In practise it appears that this is no serious restriction as is does not impede accurate computations. In Section 4 we see that even for the approximation of the singular part of the solution sufficient accuracy 
can be obtained. Assuming that $\Delta t_{i}>\Delta t_{i+1}$ (see Figure 3.1(b)), we obtain

$$
\begin{aligned}
& \left(A_{i, i+1}^{S}\right)_{k, l}=\int_{t=t_{i-1}}^{t=t_{i}} \int_{t^{\prime}=t_{i}}^{t^{\prime}=t_{i+1}} \phi_{i, k}(t) \phi_{i, l}\left(t^{\prime}\right) \frac{1}{a} I_{S}\left(\frac{\left|t-t^{\prime}\right| \mathfrak{h}_{i}}{a}, \gamma\right) d t^{\prime} d t \\
& =\int_{t=2 t_{i}-t_{i+1}}^{t=t_{i}} \int_{t^{\prime}=t_{i}}^{t^{\prime}=t_{i+1}} \phi_{i, k}(t) \phi_{i, l}\left(t^{\prime}\right) \frac{1}{a} I_{S}\left(\frac{\left|t-t^{\prime}\right| \mathfrak{h}_{i}}{a}, \gamma\right) d t^{\prime} d t \\
& =\int_{t=2 t_{i}-t_{i+1}}^{t=t_{i}} \int_{t^{\prime}=t_{i}}^{t^{\prime}=t_{i+1}-t_{i}+t} \phi_{i, k}(t) \phi_{i, l}\left(t^{\prime}\right) \frac{1}{a} I_{S}\left(\frac{\left|t-t^{\prime}\right| \mathfrak{h}_{i}}{a}, \gamma\right) d t^{\prime} d t \\
& \approx \int_{\tau=-1}^{0} \int_{\tau^{\prime}=0}^{1+\tau} \varphi_{k}(\tau+1) \varphi_{l}\left(\tau^{\prime}\right) \frac{\Delta t_{i} \Delta t_{i+1}}{a} \times \\
& I_{S}\left(\frac{\left|\tau-\tau^{\prime}\right| \mathfrak{h}_{i} \sqrt{\Delta t_{i} \Delta t_{i+1}}}{a}, \gamma\right) d \tau^{\prime} d \tau \\
& =\frac{\Delta t_{i} \Delta t_{i+1}}{a} \int_{\xi=\frac{-1}{\sqrt{2}}}^{0} \int_{\eta=\xi}^{-\xi} \varphi_{k}\left(\frac{\eta+\xi}{\sqrt{2}}+1\right) \varphi_{l}\left(\frac{\eta-\xi}{\sqrt{2}}\right) \times \\
& I_{S}\left(\frac{\sqrt{2}|\xi| \mathfrak{h}_{i} \sqrt{\Delta t_{i} \Delta t_{i+1}}}{a}, \gamma\right) d \eta d \xi \\
& =\frac{\Delta t_{i} \Delta t_{i+1}}{a} \int_{\xi=\frac{-1}{\sqrt{2}}}^{0} I_{S}\left(\frac{\sqrt{2}|\xi| \mathfrak{h}_{i} \sqrt{\Delta t_{i} \Delta t_{i+1}}}{a}, \gamma\right) \widehat{\mathcal{Q}}_{k, l}(-\xi) d \xi \\
& =\frac{\Delta t_{i} \Delta t_{i+1}}{a} \int_{\xi=-1}^{0} I_{S}\left(\frac{|\xi| \mathfrak{h}_{i} \sqrt{\Delta t_{i} \Delta t_{i+1}}}{a}, \gamma\right) \frac{\widehat{\mathcal{Q}}_{k, l}(-\xi / \sqrt{2})}{\sqrt{2}} d \xi \\
& =\frac{\Delta t_{i} \Delta t_{i+1}}{a} \overline{\mathcal{I}}_{k, l}\left(\frac{\mathfrak{h}_{i} \sqrt{\Delta t_{i} \Delta t_{i+1}}}{a}, \gamma\right) \text {, }
\end{aligned}
$$

where we have introduced

$$
\begin{aligned}
\widehat{\mathcal{Q}}_{k, l}(\xi) & =\int_{\eta=-\xi}^{\xi} \varphi_{k}\left(\frac{\eta-\xi}{\sqrt{2}}+1\right) \varphi_{l}\left(\frac{\eta+\xi}{\sqrt{2}}\right) \\
\overline{\mathcal{Q}}_{k, l}(\xi) & =\frac{1}{\sqrt{2}} \widehat{\mathcal{Q}}_{k, l}\left(\frac{\xi}{\sqrt{2}}\right) \\
\overline{\mathcal{I}}_{k, l}(x, \gamma) & =\int_{\xi=0}^{1} \overline{\mathcal{Q}}_{k l}(\xi) I_{S}(\xi x, \gamma) d \xi
\end{aligned}
$$

In case that $\Delta t_{i}<\Delta t_{i+1}$ (Figure 3.1(c)), identical results are obtained. Similarly, we find

$$
\left(B_{i, i+1}\right)_{k, l} \approx \frac{1}{a}{\overline{\mathcal{I} \mathcal{Q}^{\prime}}}_{k, l}\left(\frac{\mathfrak{h}_{i} \sqrt{\Delta t_{i} \Delta t_{i+1}}}{a}, \gamma\right)
$$


where we have introduced

$$
\begin{aligned}
& \widehat{\mathcal{Q}}_{k, l}^{\prime}(\xi)=\int_{\eta=-\xi}^{\xi} \varphi_{k}^{\prime}\left(\frac{\eta-\xi}{\sqrt{2}}+1\right) \varphi_{l}^{\prime}\left(\frac{\eta+\xi}{\sqrt{2}}\right), \\
& {\overline{\mathcal{Q}^{\prime}}}_{k, l}(\xi)=\frac{1}{\sqrt{2}}{\widehat{\mathcal{Q}^{\prime}}}_{k, l}\left(\frac{\xi}{\sqrt{2}}\right) \text {, } \\
& {\overline{\mathcal{I} \mathcal{Q}^{\prime}}}_{k, l}(x, \gamma)=\int_{\xi=0}^{1} \overline{\mathcal{Q}}_{k l}^{\prime}(\xi) I_{S}(\xi x, \gamma) d \xi \text {. }
\end{aligned}
$$

Although these are somewhat complex expressions, the functions $\widehat{\mathcal{Q}}_{k, l}(\xi), \overline{\mathcal{Q}}_{k, l}(\xi)$, $\overline{\mathcal{I}}_{k, l}(x, \gamma)$ and their primed counterparts are easily precomputed once and for all.

3.4. The $h p$-adaptive strategy. Our $h p$-adaptive strategy is an iterative procedure that starts with a coarse uniform mesh on which a low order $(p=2)$ solution is computed. In each step of the iteration it is decided, for each element, whether it should be $h$-refined (i.e., the element is split into two equal smaller elements), or $p$-refined (i.e., the order of the approximation in that element is enhanced by one order). If it is decided that the approximation is locally accurate enough no further refinement is applied in that element. The procedure ends when no element requires any further refinement or, for the elements that still do, the element-size drops below some given minimal value.

The decision on whether to apply $p$ - or $h$-refinement is made on basis of the local smoothness of the computed solution. The smoothness of the solution is determined from the behaviour of the coefficients in the hierarchical representation of the solution in an element. As explained in Section 2.3, for a sufficiently smooth solution we may expect the coefficients to decrease geometrically if the element-size is small enough. Therefore, we consider in each element the sequence of coefficients as found in (2.16), i.e., $\left|c_{p}+c_{0}\right| / 2,\left|c_{p}-c_{0}\right| / 2,\left|c_{1}\right|,\left|c_{2}\right|, \cdots,\left|c_{p-1}\right|$. In particular the tail of this sequence, (i.e., the last 3 available coefficents) is used to determine the smoothness.

Denoting the coefficients in the tail by respectively $C_{1}, C_{2}$ and $C_{3}$, and introducing an decrement factor $\kappa>1$, we compare the values of the triple $\left\{C_{1}, \kappa C_{2}, \kappa^{2} C_{3}\right\}$, distinguishing 2 situations:

- the tail is decreasing if $\kappa^{2} C_{3}<C_{1}$, or

- the tail is increasing if $\kappa^{2} C_{3} \geq C_{1}$.

In case of an increasing tail, the triple can be

- monotonous, or

- of V-type, i.e., $\kappa C_{2}<\min \left(C_{1}, \kappa^{2} C_{3}\right)$, or

- of A-type, i.e., $\kappa C_{2} \geq \max \left(C_{1}, \kappa^{2} C_{3}\right)$.

The decision on $p$ - or $h$-refinement is now made as follows:

1. if the coefficient tail is decreasing we qualify the solution to be locally smooth, and we take the last computed coefficient in the hierarchical basis, $C_{3}$, as the current local error. If this error is larger than a prescribed tolerance, we decide for $p$-refinement;

2. if the coefficient tail is monotonously increasing or of increasing $V$-type, then adding the last correction proved to have an adverse effect. The solution is qualified as non-smooth, motivating to $h$-refine the element. $C_{2}$ is taken as the local error. In the two new elements we use the same order $p$ as was used in the original unsplit element.

3. if the coefficient tail is of increasing A-type, then the previous triple in the same element can only have been of $\mathrm{V}$-type. This means that the coefficient 
$C_{1}$ is (possibly by coincidence) relatively small. We consider adding the correction corresponding to $C_{3}$ to be beneficial, the solution to be locally smooth and we take $C_{3}$ as the local error. If this local element is larger than a prescribed tolerance, we decide to $p$-refine the element.

In this way we control the $L^{\infty}$-error on each element. We notice that a larger factor $\kappa$ requires a faster decreasing sequence of hierarchical coefficients, and hence introduces a bias towards $h$-refinement, whereas a smaller $\kappa>1$ leads to more $p$-refinement. In this work we use $\kappa=4$ as it gives a good balance between $h$-and $p$-adaption as shown Section 4.

The mesh refinement iterative process requires at every iteration the elementby-element assembly of the discrete operator. The hierarchical basis is such that the elementary operator of a $p$-refined element equals the previously computed one augmented by one row and column. We exploit this fact in our simulation code by remembering previously computed results.

Stopping Criterium. Motivated by our arguments in Section 3.2, we tailor the adaptive refinement process so as to ensure that the discretization error $\triangle V$ in the voltage computation is smaller than a prescribed tolerance TOL. Because of (1.12), we see that $\triangle V \leq C\|\Delta I(t)\|_{L^{1}(\Omega)}$, where $\Delta I(t)$ denotes the error in $I(t)$. On a mesh consisting of $N$ elements we allow on each element an $L^{1}$-error of TOL $/ N$, which implies an $L^{\infty}$-error of TOL $/(N h)$. In this way we distribute the $L^{1}$-error over the grid, allowing for a larger $L^{\infty}$-error on smaller elements.

Numerical evidence given in Section 4.3 shows that starting computations with a mild tolerance and using the final mesh obtained as point of departure for a more accurate computation results in a slower increase of the degrees of freedom than when requiring a stringent tolerance from the start.

4. Numerical Results. In this section we describe four examples. First we show how the $h p$-refinement strategy works for the computation of the current in a receiving straight wire antenna. Next we do the same for an emitting antenna. In the third example we study how the accuracy requirement for the induced voltage across a gap in the receiving antenna influences the required number of degrees of freedom. For comparison with results from other papers, in these first three examples we still consider the classical straight thin-wire antenna. In the last example we perturb the geometry of the antenna and employ the method developed to track the effect of the curvature on the induced voltage.

4.1. Current in Receiving Antenna. In the first experiment, we consider the computation of the current in a straight wire antenna of length $L=1 \mathrm{~m}$ and radius $a=0.001 \mathrm{~m}$, induced by a plane wave exitation of frequency $f=500 \mathrm{MHz}$ and propagation vector perpendicular to the axis of the antenna. We use the $L^{1}$-error bound introduced in Section 3.4 as the stopping criterium in the mesh refinement process. As initial discretization we choose a uniform mesh with 16 second degree elements having in total 33 (i.e., $17+16$ ) degrees of freedom. Imposing a tolerance $\mathrm{TOL}=5 e-8 \mathrm{Am}$, this results in eight adaptive refinement steps and a mesh of 96 elements with 422 degrees of freedom. In Figure 4.1(a) for the final mesh we plot the number of local mesh refinements and polymomial order. In Figure 4.1(b) we show the computed current. The mesh shows to have been $h$-refined near the end-point singularities and to have been $p$-refined where the current is a smooth function. The behavior of the $L^{1}$-norm of the error in the computed current as a function of the mesh refinement step in shown in Figure 4.3(a). On the final mesh the estimated $L^{\infty}$-error in the current is $8.47 e-7 \mathrm{~A}$. 
4.2. Current in Emitting Antenna. This time we consider the computation of the current in an emitting straight antenna. Length and radius are as in the previous example. Now the antenna has a gap of $0.01 \mathrm{~m}$ in its middle where a current source of $1 \mathrm{~A}$ is impressed. Starting from a piecewise second degree solution on a uniform mesh with eight elements on either side of the gap and imposing a tolerance TOL $=1 e-3 \mathrm{Am}$, seven adaptive refinement steps result in a mesh of 45 elements with 154 degrees of freedom. The final $h p$-mesh and computed current are shown in Figure 4.2. Both the end-point singularities and the singularities at the boundaries of the gap are clearly resolved by low order small elements. The convergence history of the $L^{1}$-norm of the error in the current is shown in Figure 4.3(b). The estimated $L^{\infty}$-error in the computed current is $1.64 e-2 \mathrm{~A}$.

4.3. Voltage Across a Gap in Straight Wire. Now we compute the voltage induced over a gap in a receiving straight wire antenna excited by a plane wave at $500 \mathrm{MHz}$ with propagation vector perpendicular to the antenna axis. The values for $L, a$, the gap size and the initial mesh are the same as above. We investigate how the required tolerance affects the following three quantities considered as functions of the index of the mesh refinement step: the number of degrees of freedom, the error in the amplitude of the voltage and the $L^{1}$-norm of the error in the current. On each mesh, the error in the voltage amplitude is computed by considering the error in the current on an element to be given by the highest degree basis function times the corresponding coefficient, substituting this error in (1.12) and taking the absolute value of the result. We illustrate in particular how a stepwise reduction of the tolerance results in a savings of the degrees of freedom. In the top row of Figure 4.4 we show the behavior of the three functions of interest for three different values of TOL $(\mathrm{TOL}=1 e-2,1 e-3,1 e-4 \mathrm{Am})$ starting from a piecewise second degree solution on a uniform mesh with four (as opposed to eight in the previous example) elements on either side of the gap. In the last case, nine refinement steps result in a mesh with 608 degrees of freedom, an estimated $L^{\infty}$-error in the computed current of $6.48 e-4 \mathrm{~A}$ and an estimated error in the amplitude of the voltage of $3 e-6 \mathrm{~V}$. In the bottom row of Figure 4.4 we plot the convergence history of the same three quantities starting with $\mathrm{TOL}=1 e-2 \mathrm{Am}$, stepwise reducing this value to TOL $=1 e-3 \mathrm{Am}$ after the fourth iteration and TOL $=1 e-4 \mathrm{Am}$ after the ninth iteration, respectively. In this way 14 iterations results in a mesh of 516 degrees on freedom. This is a significant reduction compared with the 608 degrees of freedom generated before.

4.4. The Effect of Perturbations in the Geometry of the Wire. In this last example we perturb the straight wire by a sinus profile, setting the functions $h_{1}(t)$ and $h_{2}(t)$ introduced in Section 1.1 equal to $h_{1}(t)=0$ and $h_{2}(t)=w L \sin (\pi t)$ for $0 \leq t \leq L$, respectively (see Figure 4.5(a)): we study how the induced voltage changes with $w$ for $w$ ranging between 0 and 1.5. The values for $L$ and $a$, the location and width of the gap, as well as the frequency of the excitation are chosen as in the previous examples. The progation vector is assumed to be perpendicular to the $x$ axis. In the range of $w$ considered, the total length of the wire varies between $1 \mathrm{~m}$ and approximately $3.17 \mathrm{~m}$ and is thus equal to an integer multiple of the the wavelenght $\lambda=0.6 \mathrm{~m}$ at four instances. In Figure 4.5(b) we plot the amplitude of the voltage $|V(w)|$ as a function of $w$. Four resonances can be clearly distinguished.

5. Conclusion. In this paper we solve Pocklington's equation with exact kernel for the voltage induced across gaps in arbitrarily curved thin wire antennas. For the efficient discretization we introduced a new adaptive refinement strategy. The key is 


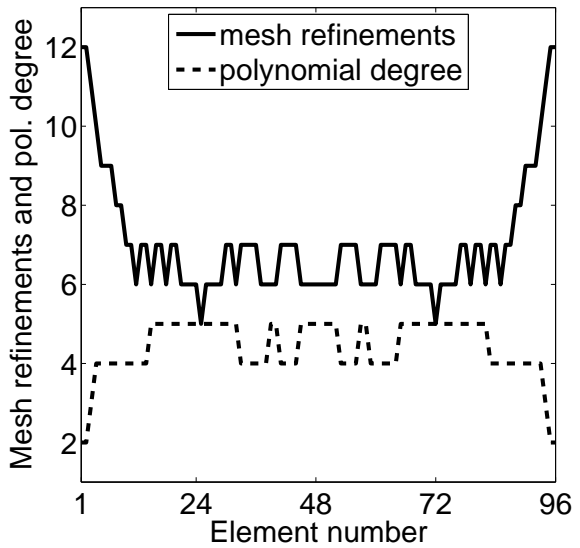

(a) Final mesh

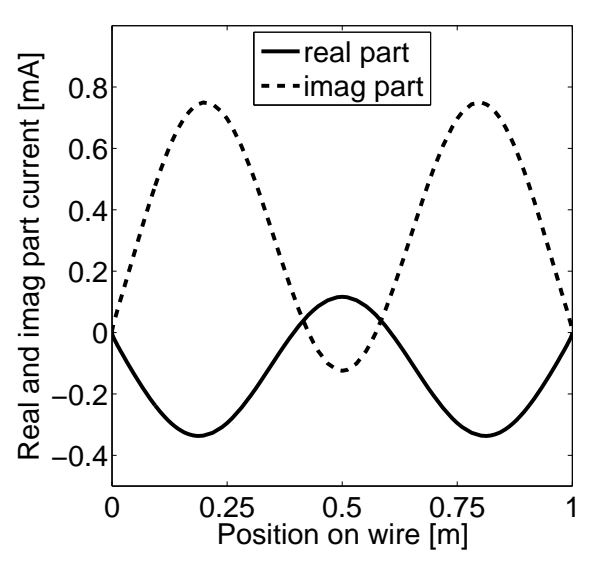

(b) Computed current

FIG. 4.1. Results for a receiving straight wire antenna. Left: the number of mesh refinement steps and the polynomial degree yielding an $L^{1}$-error smaller than $T O L=5 e-8 \mathrm{Am}$. Right: the real and imaginary part of the computed current.

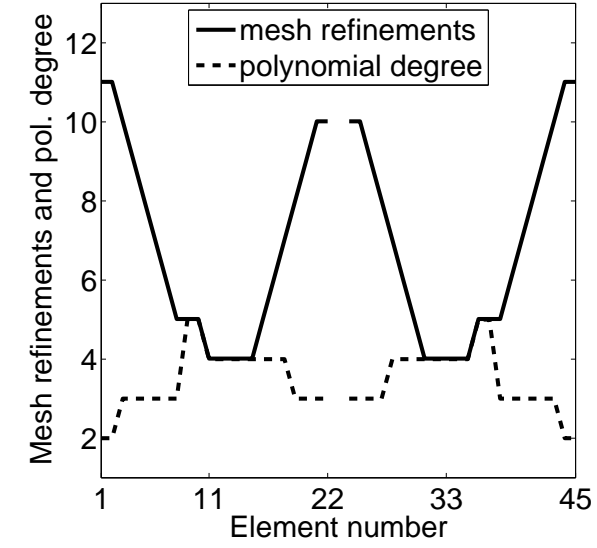

(a) Final mesh

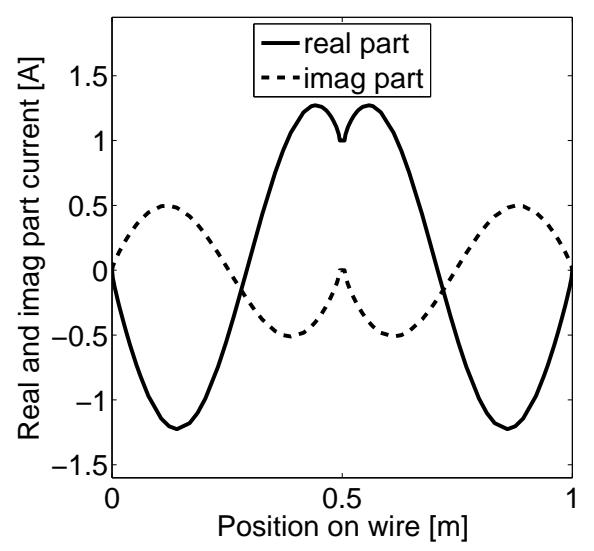

(b) Computed current

FIG. 4.2. Results for an emitting straight wire antenna. Left: the number of mesh refinement steps and the polynomial degree yielding an $L^{1}$-error smaller than $T O L=3 e-3 \mathrm{Am}$. Right: the real and imaginary part of the computed current.

that the degree of local smoothness of the solution can be derived from piecewise $p$ hierarchical basis coefficients. This information is used to decide whether a particular element has to be $h$ - or $p$-refined. Numerical results for straight and curved antennas demonstrate both the simplicity and efficiency of the approach.

\section{REFERENCES}

[1] M. Abramowitz And A. S. Stegun, eds., Handbook of Mathematical Functions, Dover, New York, 1992. Reprint of the 1972 edition.

[2] G. J. Burke, Numerical Electromagnetics Code - NEC-4 Method of Moments, Part II, Program Description - Theory, Tech. Report UCRL-MA-109833, Lawrence Livermore National 


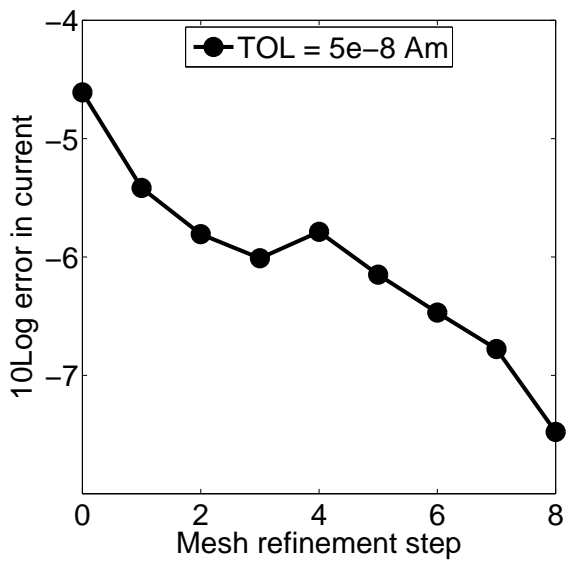

(a) Receiving antenna

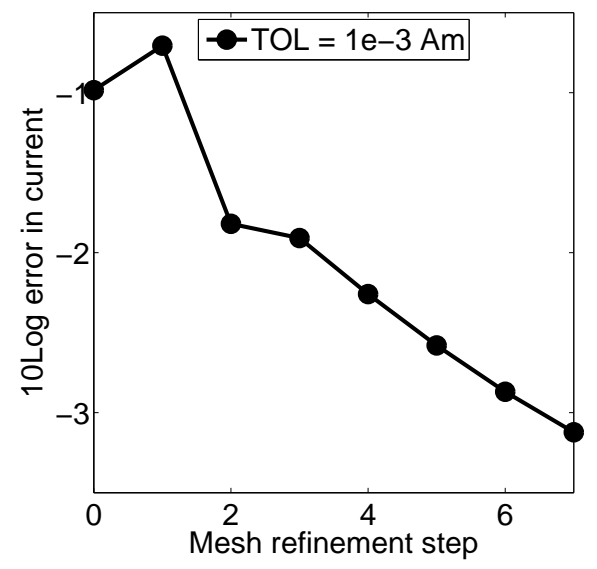

(b) Emitting antenna

FIG. 4.3. Convergence history of the mesh adaption process for the receiving (left) and emitting (right) antenna. The graph show the behavior of the $L^{1}$-norm of the error in the current as function of the mesh refinement step.

Laboratory, 1992.

[3] C. Carstensen and B. P. Rynne, A Posteriori Error Control for the Finite Element Approximations of the Integral Equation for Thin Wire Antennas, Z. Angew. Math. Mech., 82 (2002), pp. 284-288.

[4] P. J. Davies, D. B. Duncan, And S. A. Funken, Accurate and Efficient Algorithms for Frequency Domain Scattering from a Thin Wire, J. Computational Phyiscs, 168 (2001), pp. $155-183$.

[5] A.T. DE Hoop, The N-port Receiving Antenna and its Equivalent Network, in Philips Research Reports (Special issue in honor of C. Bouwkamp), no. 30, 1974, pp. 302-315.

[6] J. Goedbloed, Reciprocity and emc measurements, IEEE EMC EMCS Newsletter, (2003), pp. $93-104$.

[7] D. S. Jones, The Theory of Electromagnetism, International Series of Monographs on Pure and Applied Mathematics, Pergamon Press, 1964.

[8] - Note on the Integral Equation for a Thin Straight Wire, IEE Proc., 128 (1981), pp. 114116.

[9] K. K. Mei, On the Integral Equations of Thin Wire Antennas, IEEE Trans. Antennas Propagat., 13 (1965), pp. 374-378.

[10] B. L. Michielsen, A New Approach to Electromagnetic Shielding, in Proc. Int. Zurich EMC Symposium, 1985, pp. 509-514.

[11] L. PeArson, A Seperation of the Logarithmic Singularity in the Exact Kernel of the Cylindrical Antenna Integral Equation, IEEE Trans. Antennas Propagat., 23 (1975), pp. 256-258.

[12] H. C. Pocklington, Electrical Oscillations in Wires, in Proceedings of the Cambridge Philosphical Society, vol. 9, 1897, pp. 324-332.

[13] B. P. Rynne, The Well-Posedness of the Integral Equations for Thin Wire Antennas, IMA J. Applied Math., 49 (1992), pp. 35-44.

[14] - Convergence of Galerkin Method Solutions of the Integral Equation of Thin Wire Antennas, Ad. Comp. Math., 12 (2000), pp. 251-259.

[15] A. G. Tiuhuis, P. Zhongqiu, and A. R. Bretones, Transient Exitation of a Straight-Wire Segment: a New Look to an Old Problem, IEEE Trans. Antennas Propagat., 40 (1992), pp. $1132-1146$.

[16] M. C. van Beurden And A. G. Tijhuis, Analysis and Regularization of the Thin-Wire Integral Equation With Reduced Kernel, IEEE Trans. Antennas Propagat., 55 (2007), pp. 120-129.

[17] D. H. Werner, Huffman J. A., And P. L. Werner, Techniques for Evaluating the Uniform Current Vector Potential at the Isolated Singularity of the Cylindrical Wire Kernel, IEEE Trans. Antennas Propagat., 42 (1994), pp. 1549-1153.

[18] H. Yserentant, Hierarchical basis, in Proceedings of the Second International Conference on Industrial and Applied Mathematics (ICIAM 91), R.E. O'Malley, ed., 1992, pp. 256-276. 


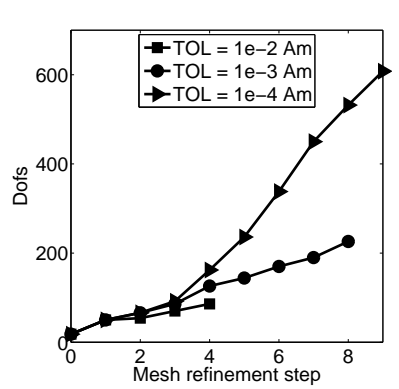

(a) Degrees of freedom

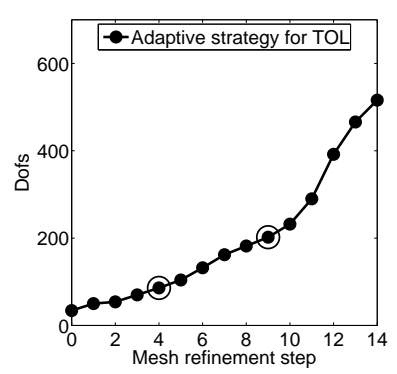

(d) Degrees of freedom

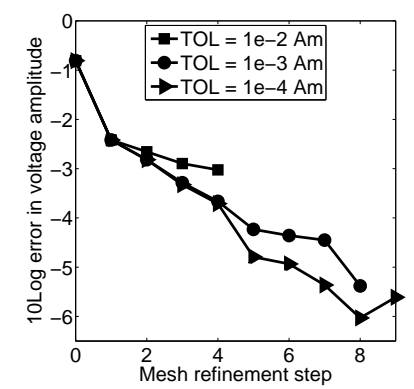

(b) Correction in voltage

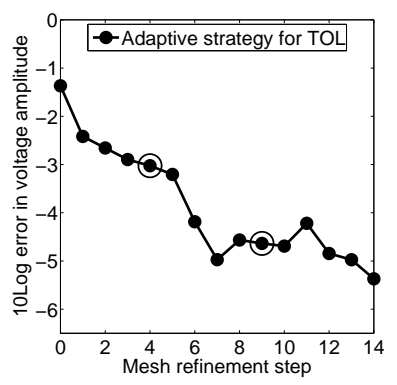

(e) Correction in voltage

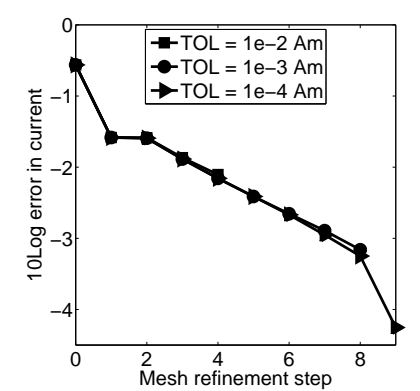

(c) Error in current

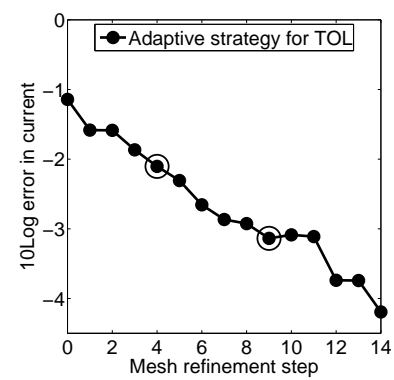

(f) Error in current

FIG. 4.4. Top row: number of degrees of freedom, error in amplitude of voltage and $L^{1}$ norm of the error in the current as functions of the mesh refinement step for TOL $=1 \mathrm{e}-2 \mathrm{Am}$, $T O L=1 e-3 \mathrm{Am}$ and $T O L=1 e-4 \mathrm{Am}$. Bottom row: adaptive strategy for TOL, decreasing its value during the iteration (starting with $T O L=1 e-2 \mathrm{Am}$, after the fourth iteration $T O L=1 e-3 \mathrm{Am}$ and after the ninth iteration $T O L=1 e-4 \mathrm{Am}$ ).

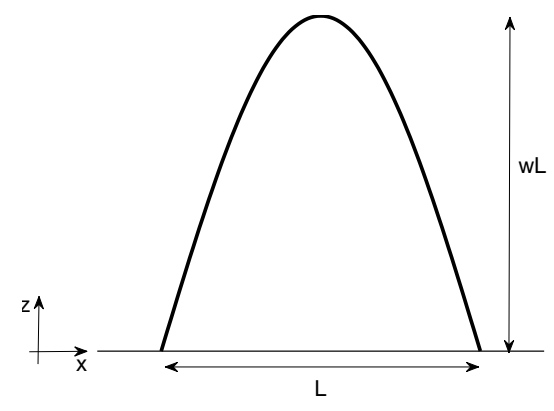

(a) Geometry of the wire.

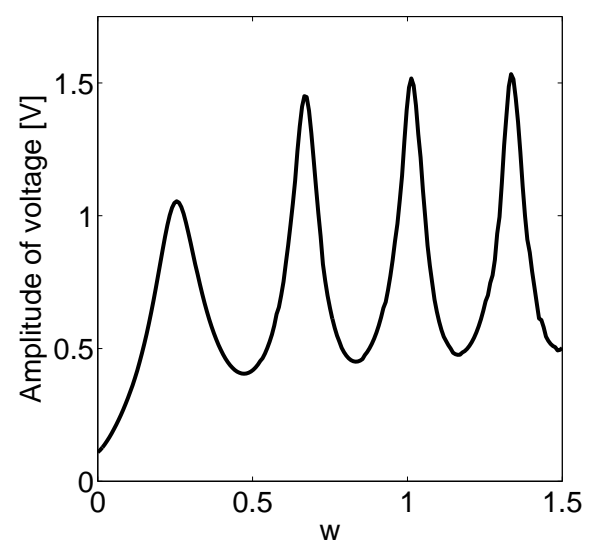

(b) Amplitude of the induced voltage.

FIG. 4.5. Results for a curved receiving wire antenna. Left: geometry of the wire. Right: Amplitude of the induced voltage as a function of the amplitude of the perturbation. 